\title{
Reproductive migrations of the sex role reversed pipefish Nerophis lumbriciformis (Pisces; Syngnathidae)
}

\author{
N. M. Monteiro†t, A. Berglund $\S, M \cdot$ N. Vieira*† \\ And V. C. Almada 9 \\ *Departamento de Zoologia e Antropologia da Faculdade de Ciências da \\ Universidade do Porto, Praça Gomes Teixeira, 4099-002 Porto, Portugal, \\ $\uparrow$ CIIMAR, Rua dos Bragas 177, 4050-123 Porto, Portugal, §Department of Animal \\ Ecology, Norbyv. 18d, Uppsala University, SE-752 36 Uppsala, Sweden and $₫ I S P A$, \\ Rua Jardim do Tabaco 34, 1149-041 Lisboa, Portugal
}

(Received 22 February 2005, Accepted 27 December 2005)

\begin{abstract}
Using an individual identification technique, a population of worm pipefish Nerophis lumbriciformis was followed during 19 months, in order to determine the exact use of the intertidal and, considering the specific movement patterns of males and females, the mating system exhibited by this population. Field observations showed that the number of adults increased during the breeding season, with males arriving 1 month earlier than females. Furthermore, males and females presented distinct permanence periods, showing that the intertidal is used as a mating arena. It was also observed that both male and female worm pipefish mated repeatedly over the span of a reproductive season, but females exhibited shorter remating intervals. Also, females stayed for longer periods on the mating grounds, the intertidal zone, whereas males typically left for the subtidal after mating, usually returning within 2 months. These inter-sexual differences in the occupation of the intertidal suggest that females breed with different males but also that males accept eggs from various females since, on their return, a new group of mating partners was now available. Thus, $N$. lumbriciformis might be considered polygynandric. It is a clearly dimorphic species in spite of the observed polygynandry, suggesting that differences in remating intervals may be influential in determining the strength of sexual selection along with what may be expected from the polygynandrous mating system alone.

(C) 2006 The Authors

Journal compilation $\odot 2006$ The Fisheries Society of the British Isles
\end{abstract}

Key words: mating system; Nerophis lumbriciformis; polygynandry; potential reproductive rate; Syngnathidae.

\section{INTRODUCTION}

The reproductive biology of the Syngnathidae (pipefishes, seahorses and seadragons) is characterized by one of the most specialized forms of parental care, male pregnancy. Females deposit eggs in a specialized incubating area, located either on the abdomen or tail of the male. Embryos then undergo a more

\$Author to whom correspondence should be addressed at present address: Faculdade de Ciências da Saúde, Universidade Fernando Pessoa, Rua Carlos da Maia, 296, 4050-123 Porto, Portugal. Tel.: +351 22 3401514; fax: +351 22 3401511; email: nmonteir@fc.up.pt 
or less prolonged incubation period (Herald, 1959). The evolutionary radiation of this family was accompanied by a diversification of structures involved in parental care (Wilson et al., 2003) and various degrees of brood pouch complexity are still visible within the Syngnathidae. One of the simplest brooding structures, probably similar to that of the ancestral syngnathid (Wilson et al., 2001), can still be observed in the marsupium-lacking genera Entelurus and Nerophis. Part of one of the most basal lineages (Herald, 1959; Wilson et al., 2001), these genera are important for the understanding of the evolution of sex-role reversal and the relationship between the mating system and degree of sexual dimorphism.

The worm pipefish Nerophis lumbriciformis (Jenyns), is amongst the smallest of the western European pipefishes. It can commonly be found in rocky shores from the intertidal to c. $30 \mathrm{~m}$ depth (Dawson, 1986; Wheeler, 1969), mainly among seaweeds or under boulders (Monteiro et al., 2002a). Its distribution ranges from the Atlantic coast of Norway to the Kattegat, and from Belgium southwards to Morocco (Dawson, 1986). It feeds on small vagile epiphytic crustaceans (Lyons \& Dunne, 2004), 'sucking' them through its characteristically upwards pointing snout. During the breeding season, males brood their offspring attached to their flattened ventral surface (Monteiro et al., 2002b). Immediately after hatching, the newborn are free-swimming, planktonic, and no further care is provided (Monteiro et al., 2003).

Following similar patterns as those observed in Nerophis ophidion (L.) (Berglund et al., 1986a, 1989; Rosenqvist, 1990), field and laboratory studies showed that $N$. lumbriciformis females actively compete for access to mates and possess conspicuous secondary sexual characters (Monteiro et al., 2002b, 2005). Worm pipefish females are larger and more brightly coloured, more active during courtship, and present an appearance-enlarging keel-like structure in the abdominal area, in contrast to males. Thus, given all behavioural and morphological similarities to $N$. ophidion, it is possible that $N$. lumbriciformis mating system may also tend towards polyandry (McCoy et al., 2001).

The goal of this study which used an individual identification technique was: (1) to determine the exact use of the intertidal, since this area is only occupied during part of the year by adult individuals, (2) to verify if the movement patterns of males and females in and out of the intertidal can help elucidate the species mating system and (3) to observe if the mating system and the degree of sex role reversal are in accordance with theoretical suggestions (Jones \& Avise, 2001).

\section{MATERIAL AND METHODS}

Data were collected in an intertidal rocky shore area, in Viana do Castelo $\left(41^{\circ} 41^{\prime} \mathrm{N}\right.$; $8^{\circ} 50^{\prime} \mathrm{W}$ ), Portugal, from June 2002 to December 2003. Prior to the sampling, several metallic marks were installed in the study area $\left(c .180 \mathrm{~m}^{2}\right)$, with the help of a pneumatic rotary hammer, thus acting as fixed co-ordinates. The distances between these points were registered in order to triangulate the exact position of every captured fish in the study area.

During sampling, that usually occurred monthly on low spring tides, adult fish were captured by hand, after a thorough search in rockpools, crevices and underneath boulders. Fish were measured for total length $\left(L_{\mathrm{T}}\right)$ and digitally photographed on both 
sides of the head and the location of each individual in the intertidal recorded. After the sampling process, fish were returned to their original position and stones were rearranged back to the original configuration. The comparison of the photographs of each fish with those taken during previous visits allowed for the determination of recaptures. In the case of recaptured animals, the distances between the new location and that of the previous capture were computed. In a previous study (Monteiro et al., 2005) it was demonstrated that $N$. lumbriciformis adults have natural markings that are sufficiently specific and stable to allow for individual identification. Details of the identification procedure and sampling procedures are given by Monteiro et al. (2005).

Data from previous sampling years (November 1997 to November 1999) were pooled to the data gathered during the sampling period referred to above [e.g. number of eggs per male (Monteiro et al., 2001, 2003), number of males and females arriving at the intertidal]. A one-way ANOVA was conducted in order to detect differences in the average number of eggs males carried during the onset (October to December), middle (February to May) or end (July and August) of the breeding season [three levels $(n=7$ months per level); all test assumptions were met; normality: Shapiro-Wilk $W=0 \cdot 95$, $P=0 \cdot 25$; homogeneity of variances, Cochran's $C=0 \cdot 61, P=0 \cdot 22]$. In order to check if males and females had different return rates to the intertidal, a $\chi^{2}$ independence test was used. All statistical analyses were conducted using Statistica 6.1 (StatSoft).

\section{RESULTS}

The average number of eggs per breeding male was $c .51$ ( $n=165$ males; mean \pm S.D. $=50 \cdot 83 \pm 12 \cdot 49 ;$ range $=4-84)$ and there was no correlation between the number of eggs and male size [product moment correlation; $n=165$ males; $r=0 \cdot 07 ; P=0 \cdot 41$, Fig. 1 (a)]. These results still stand even if only data from 2002 to 2003 are used $(n=51$ males; $r=0 \cdot 16 ; P=0 \cdot 256)$.

The mean number of eggs per male increased during the last months of the breeding period [one-way ANOVA, $F_{2,21}, P<0 \cdot 01$; data from 107 different males were used, see Fig. 1(b)], when compared to the onset and middle of the reproductive season (Newman-Keuls post hoc test). Additional evidence for the fact that an increase in the mean number of eggs towards the end of the breeding season is not due to a shortage of males is provided by the lack of a correlation between the percentage of pregnant males and the mean number of eggs per male ( $n=34$ months; $r=0 \cdot 14, P=0 \cdot 43$ ).

The peak of the breeding season coincided with a greater number of adults in the intertidal area, males as well as females, whereas the lowest number of adults was recorded in the period between December and February [Fig. 1(d)]. The number of captured males and females was significantly correlated throughout the years $(n=34$ months; $r=0.54 ; P<0.01)$, but there was a significant deviation from an even sex-ratio ( $t$-test of means against an 'even sex-ratio' value of $1 ; n=34$ months, $P<0 \cdot 01$ ), with a surplus of males especially noticeable during the beginning of the breeding season [Fig. 1(c)].

Even though $N$. lumbriciformis presented a strong homing behaviour, with individuals being always found $<2 \mathrm{~m}$ of the previous recapture, the periodicity of recaptures (time lag between recaptures) was different between males and females, with females typically being recaptured within the first month, while males were recaptured with longer time intervals, typically after 2-4 months (Fig. 2). There was a significant difference between the number of recaptures of males and females when time lags of 1 month were compared with those of $\geq 2$ months ( $\chi^{2}$ independence test, d.f. $\left.=1, P<0.05\right)$. 


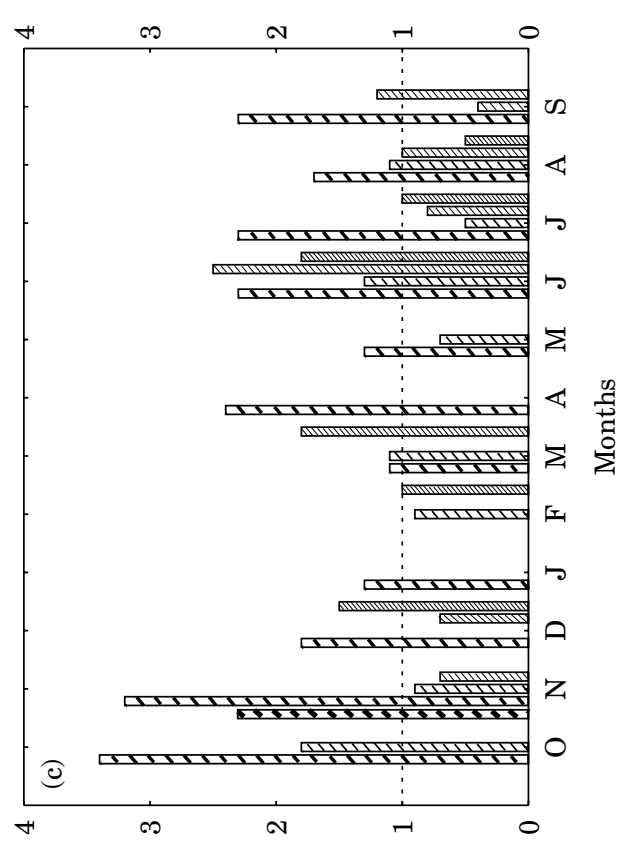

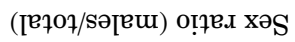
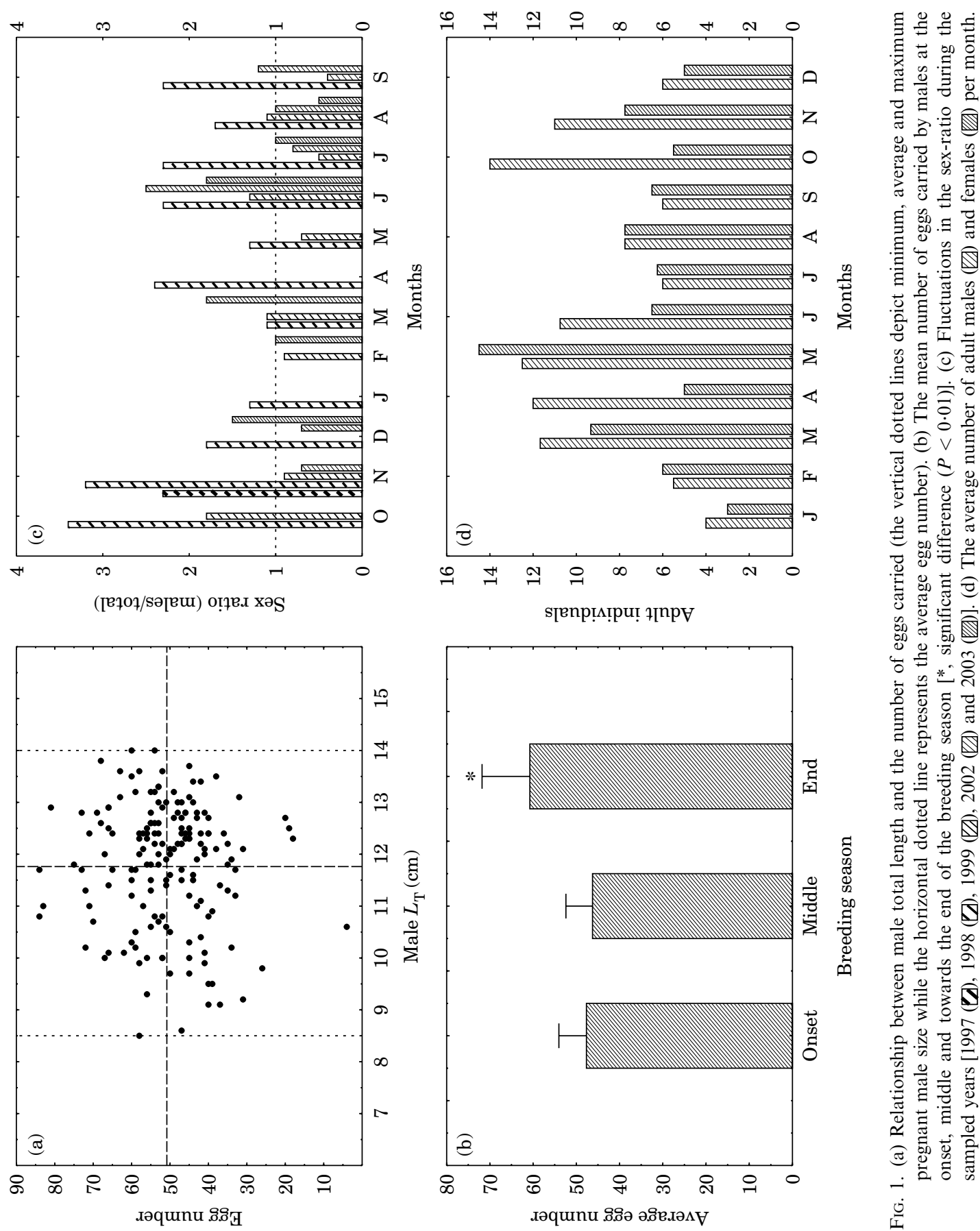


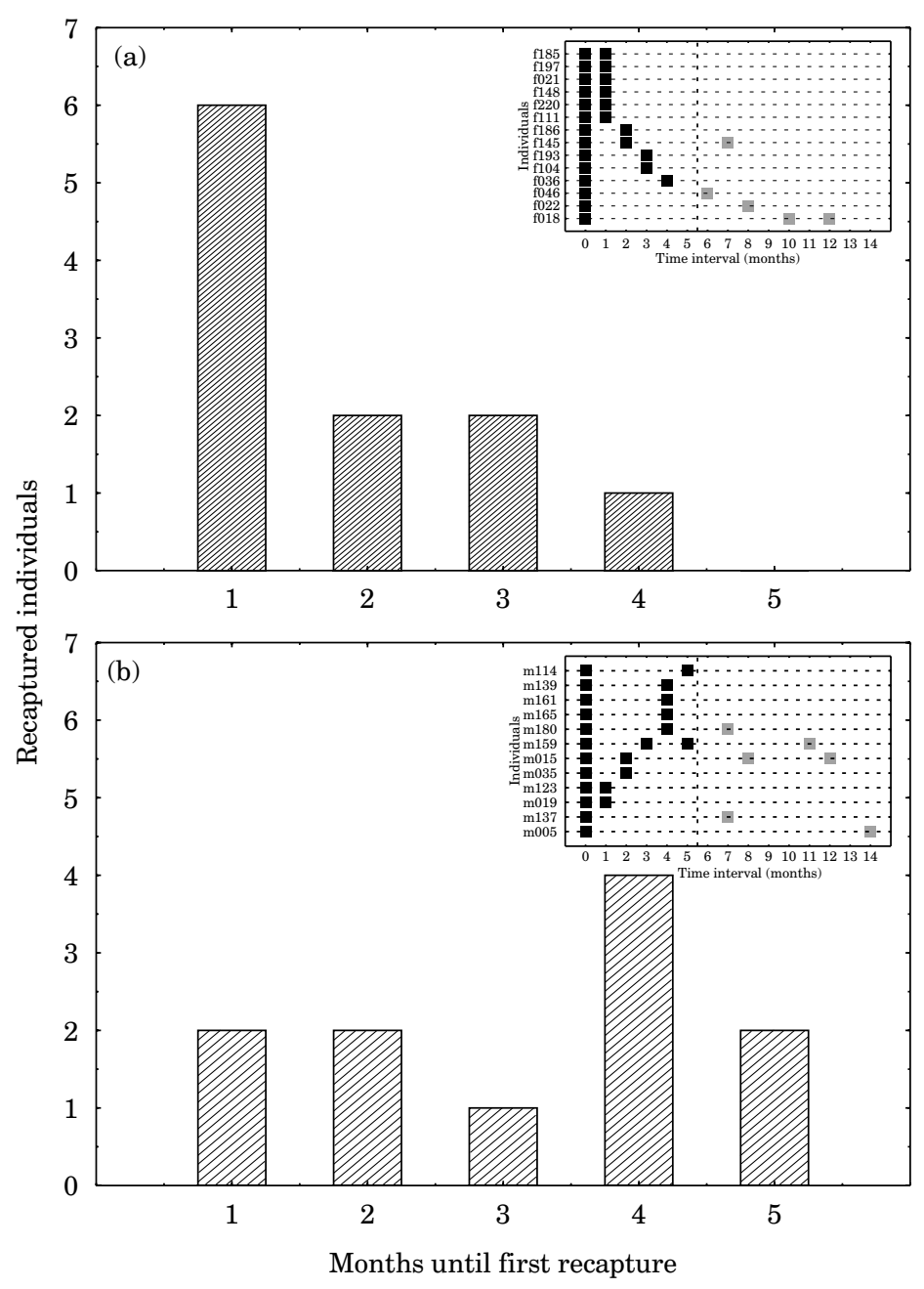

FIG. 2. Time intervals until (a) female and (b) male recaptures. Smaller graphs represent monthly intervals for specific individuals, starting at the first capture (0). recaptures that probably span two breeding seasons.

\section{DISCUSSION}

\section{THE INTERTIDAL AS A MATING ARENA}

The observation that the number of adults increased in the intertidal during the breeding season suggests that the rocky intertidal is occupied by adults during only part of the year, and that $N$. lumbriciformis is not a true intertidal resident (Monteiro et al., 2005). Additionally, males arrived c. 1 month earlier than females to the intertidal [Fig. 1(d)]. These observations suggest a migration related to reproduction. Finally, pregnant males abandoned the intertidal zone: no pregnant male was recaptured within the average pregnancy period of 30 days (Monteiro et al., 2003), with only non-pregnant males remaining (only two males 
were recaptured within 1 month and, curiously, neither were pregnant; see Fig. 2). This reinforces the notion that the intertidal is used mainly as a mating arena, rather than being an area of permanent residence.

The worm pipefish is typically found among seaweeds or underneath boulders (Monteiro et al., 2002a), so it is possible that fish migrate into the intertidal in order to mate away from potential predators in an area smaller and safer than the subtidal. Behavioural studies in aquaria showed that $N$. lumbriciformis individuals were ignored by the common intertidal fish species Lipophrys pholis (L.), Lipophrys trigloides (Valenciennes), Coryphoblennius galerita (L.), Parablennius gattorugine (L.), Lepadogaster lepadogaster purpurea (Bonnaterre) and Lepadogaster candollei Risso, even though these species recurrently showed high levels of agonistic interactions among themselves (N. M. Monteiro, unpubl. data). On the other hand, mainly subtidal species such as Gaidropsarus mediterraneus (L.), Ciliata mustela (L.) or Taurulus bubalis (Euphrasen) have been observed to prey on adult $N$. lumbriciformis or their egg clutches.

\section{MATING SYSTEM}

Even though no molecular studies have been conducted in N. lumbriciformis, males (wild or reared in aquaria) seem to carry eggs from a single female during each pregnancy, as observed also in N. ophidion (Berglund et al., 1986a, b). All pregnant males carried eggs with similar colouration and size, with embryos in the same developmental stage. Aquarium observations of several courtship events corroborate these observations, since no male was ever seen receiving eggs from more than one female. Furthermore, as observed in two marsupiumlacking pipefishes, N. ophidion (McCoy et al., 2001) and Corythoichthys haematopterus (Bleeker) (Matsumoto \& Yanagisawa, 2001), no male already carrying eggs was observed to successfully remate, a fact that might be related to the particular fertilization process of these pipefishes (Monteiro et al., 2002b) and to the need for a close contact between eggs and the parental incubating surface. The latter precludes egg deposition in multiple layers.

Data emerging both from recaptures and aquarium observations strongly suggest that females mate with different partners during one reproductive season. Aquarium observations showed that females can remate within a few days after a successful copulation and are capable of producing more eggs than a male can bear (Monteiro et al., 2002b). Hence, since females stay for longer periods in the intertidal zone (Fig. 2), they are able to sequentially mate with different males, only then abandoning the breeding area (the number of recaptured females dropped abruptly as the time lag increased). Males, on the other hand, after receiving a new clutch of eggs, undergo a 30 days pregnancy (Monteiro et al., 2003), followed by a period when the incubating surface epithelium is rearranged [observed in both $N$. lumbriciformis (N. M. Monteiro, pers. obs.) and $N$. ophidion (Carcupino et al., 2002)]. This fact, together with the observation that pregnant males seem to avoid physical contact by abandoning large concentrations of conspecifics might explain why males stay in the intertidal for shorter periods than females (Fig. 2). The observed time lags between recaptures indicate that males return after giving birth, to mate again, this time encountering a new group of mating partners since females usually stay in the intertidal for 
periods $\leq 2$ months. As an example, a particular male was repeatedly recaptured, with a time interval of at least 45 days between each recapture (far more than a pregnancy in this species) and, on all four occasions, egg clutches of different numbers were visible. No female was observed in the intertidal for $>2$ consecutive months. Thus, males as well as females may mate repeatedly with different partners within one breeding season (polygynandry), but females have a shorter remating interval than males. In other words, females have a higher potential reproductive rate than males in this species, just like in some other pipefish species (Berglund et al., 1989).

Even though the definitions of polyandry and polygynandry are intimately dependent on the time period considered for the determination of the mating system, it seems reasonable to accept that the full extent of a breeding season is an informative time frame. Otherwise, considering only the length of a male's pregnancy, it would be impossible to describe a monogamous mating system such as the one observed for several Hippocampus or Corythoichthys species (Jones et al., 1998; Matsumoto \& Yanagisawa, 2001). Thus, considering the full extent of a breeding season, similar species such as $N$. ophidion, described as polyandrous (McCoy et al., 2001), are expected to show similar mating systems as the one here described for $N$. lumbriciformis. According to Avise et al. (2002), more polyandrous species reveal greater degrees of sexual dimorphism as a result of an increased intensity in sexual selection. Nerophis lumbriciformis is a clearly dimorphic species in spite of being polygynandrous, indicating that differences in potential reproductive rates between the sexes are more influential on the strength of sexual selection than the mating system per se.

It may be expected that the number of eggs in marsupium-lacking pipefishes, such as $N$. lumbriciformis, would be dependent on male size, since the bi-dimensional incubating surface presents a limited capacity. Surprisingly, the number of eggs per breeding male was significantly higher near the end of the reproductive season. There are several possible explanations to this: either females simply have more eggs to deposit towards the end of the breeding season, or they deposit fewer than possible at the beginning. The latter is possible if females seek higher quality males at the onset of the season but become more timepressed towards the end. Alternatively, males may refrain to accept a huge clutch at the beginning of the season, possibly due to the inherent costs of a pregnancy episode (Lyons \& Dunne, 2003). Presently, these alternatives cannot be distinguished.

If females deposit fewer eggs than possible, then it could be assumed that they may not be as 'limited by males' as initially thought. Otherwise, it could be predicted that females would systematically maximize the number of transferred eggs during each mating, since they would not be able to predict when the next mating opportunity would come along. If so, a positive correlation between male size and their number of eggs should be observed, together with a constant number of eggs throughout the breeding season.

In summary, field observations demonstrate that both male and female worm pipefish mated repeatedly over the span of a reproductive season, but females with shorter remating intervals. Females stayed for longer periods on the mating grounds, the intertidal zone, whereas males typically left for the subtidal after mating. Males arrived earlier to the mating ground, and initially carried fewer 
eggs compared to egg loads towards the end of the season. Thus, it may be concluded that the sexual difference in remating intervals may be influential in determining the strength of sexual selection along with what may be expected from the polygynandrous mating system alone.

We would like to thank everybody who helped during field work and fish individual identification, especially S. Quinteira and J. Salgueiro. N. Monteiro's participation was funded by FCT, Fundação para a Ciência e a Tecnologia (SFRH/BPD/4992/2004).

\section{References}

Avise, J. C., Jones, A. G., Walker, D. \& DeWoody, J. A. (2002). Genetic mating systems and reproductive natural histories of fishes: Lessons for ecology and evolution. Annual Review of Genetics 36, 19-45. doi: 10.1146/annurev.genet.36.030602.090831

Berglund, A., Rosenqvist, G. \& Svensson, I. (1986a). Mate choice, fecundity and sexual dimorphism in two pipefish species (Syngnathidae). Behavioural Ecology and Sociobiology 19, 301-307.

Berglund, A., Rosenqvist, G. \& Svensson, I. (1986b). Reversed sex roles and parental energy investment in zygotes of two pipefish (Syngnathidae) species. Marine Ecology Progress Series 29, 209-215.

Berglund, A., Rosenqvist, G. \& Svensson, I. (1989). Reproductive success of females limited by males in two pipefish species. American Naturalist 133, 506-516.

Carcupino, M., Baldacci, A., Mazzini, M. \& Franzoi, P. (2002). Functional significance of the male brood pouch in the reproductive strategies of pipefishes and seahorses: a morphological and ultrastructural comparative study on three anatomically different pouches. Journal of Fish Biology 61, 1465-1480. doi: 10.1111/j.10958649.2002.tb02490.x

Dawson, C. E. (1986). Syngnathidae. In Fishes of the North-eastern Atlantic and the Mediterranean (Whitehead, P. J. P., Bauchot, M. L., Hureau, J. C., Nielsen, J. \& Tortonese, E., eds), pp. 628-639. Paris: Unesco.

Herald, E. S. (1959). From pipefish to seahorse - a study of phylogenetic relationships. Proceedings of the Californian Academy of Sciences 29, 465-473.

Jones, A. G. \& Avise, J. C. (2001). Mating systems and sexual selection in male-pregnant pipefishes and seahorses: Insights from microsatellite-based studies of maternity. The Journal of Heredity 92, 150-158. doi: 10.1093/jhered/92.2.150

Jones, A. G., Kvarnemo, C., Moore, G. I., Simmons, L. W. \& Avise, J. C. (1998). Microsatellite evidence for monogamy and sex-biased recombination in the Western Australian seahorse Hippocampus angustus. Molecular Ecology 7, 1497-1505. doi: 10.1046/j.1365-294x.1998.00481.x

Lyons, D. O. \& Dunne, J. J. (2003). Reproductive costs to male and female worm pipefish. Journal of Fish Biology 62, 767-773. doi: 10.1046/j.00221112.2003.00061.x

Lyons, D. O. \& Dunne, J. J. (2004). Inter- and intra-gender analyses of feeding ecology of the worm pipefish (Nerophis lumbriciformis). Journal of the Marine Biological Association of the United Kingdom 84, 461-464. doi: 10.1017/S0025315404009452h

Matsumoto, K. \& Yanagisawa, Y. (2001). Monogamy and sex role reversal in the pipefish Corythoichthys haematopterus. Animal Behaviour 61, 163-170. doi: $10.1006 /$ anbe. 2000.1550

McCoy, E. E., Jones, A. G. \& Avise, J. C. (2001). The genetic mating system and tests for cuckoldry in a pipefish species in which male fertilize eggs and brood offspring externally. Molecular Ecology 10, 1793-1800. doi: 10.1046/j.0962-1083. 2001.01320.x

Monteiro, N. M., Almada, V. C., Santos, A. M. \& Vieira, M. N. (2001). The breeding ecology of the pipefish Nerophis lumbriciformis and its relation to latitude and 
water temperature. Journal of the Marine Biological Association of the United Kingdom 81, 1031-1033. doi: 10.1017/S002531540100501x

Monteiro, N. M., Almada, V. C. \& Vieira, M. N. (2003). Early life history of the pipefish Nerophis lumbriciformis (Pisces: Syngnathidae). Journal of the Marine Biological Association of the United Kingdom 83, 1179-1182. doi: 10.1017/ S0025315403008452h

Monteiro, N. M., Vieira, M. N. \& Almada, V. C. (2002a). Activity rhythms and cyclical changes in microhabitat preferences in the intertidal pipefish Nerophis lumbriciformis (Pisces: Syngnathidae). Acta Ethologica 5, 39-43. doi: 10.1007/s10211-0020060-7

Monteiro, N. M., Vieira, M. N. \& Almada, V. C. (2002b). The courtship behaviour of the pipefish Nerophis lumbriciformis: reflections of and adaptation to intertidal life. Acta Ethologica 4, 109-111. doi: 10.1007/s102110100048

Monteiro, N. M., Vieira, M. N. \& Almada, V. C. (2005). Homing behaviour and individual identification of the pipefish Nerophis lumbriciformis (Pisces; Syngnathidae): a true intertidal resident? Estuarine, Coastal and Shelf Science 63, 93-99. doi: 10.1016/j.ecss.2004.10.012

Rosenqvist, G. (1990). Male mate choice and female-female competition for mates in the pipefish Nerophis ophidion. Animal Behaviour 39, 1110-1115.

Wheeler, A. (1969). The Fishes of the British Isles and Northwest Europe. London: Macmillan.

Wilson, A. B., Vincent, A., Ahnesjo, I. \& Meyer, A. (2001). Male pregnancy in seahorses and pipefishes (Family Syngnathidae): Rapid diversification of paternal brood pouch morphology inferred from a molecular phylogeny. The Journal of Heredity 92, 159-166. doi: 10.1093/jhered/92.2.159

Wilson, A. B., Ahnesjo, I., Vincent, A. C. J. \& Meyer, A. (2003). The dynamics of male brooding, mating patterns, amd sex roles in pipefishes and seahorses (Family Syngnathidae). Evolution 57, 1374-1386. 